\title{
RETOS PARA EL TRABAJO DE CAMPO, INSTRUMENTOS TRADICIONALES EN MODALIDADES ACTUALES PARA EL TRABAJO SOCIAL
}

\author{
CHALLENGES FOR FIELDWORK, TRADITIONAL INSTRUMENTS IN
} CURRENT MODALITIES TOWARDS SOCIAL WORK

\section{Susana Aurelia Preciado Jiménez ${ }^{1}$}

Resumen

\begin{abstract}
En este artículo se presenta una propuesta para la recolección de datos de fuentes primarias utilizando las herramientas que se encuentran en la red tanto en Google, como en las redes sociales. Particularmente, se describe la experiencia en el uso de tres herramientas: formularios de Google, WhatsApp y Messenger de Facebook, como modalidades para la comunicación mediada por el internet con los sujetos de investigación. Estas herramientas se utilizaron en el curso de Seminario de Investigación en la Facultad de Trabajo Social identificando su utilidad durante el proceso de investigación, demostrando que fueron útiles en la recolección, captura y análisis de la información.
\end{abstract}

Palabras claves: Recolección de Datos; Tecnologías; Trabajo Social.

Abstract

In this article, presents a proposal for the collection of data from primary sources, using the tools found in the network such as Google and social networks. In particular, we will describe the experience in using three tools: Google forms, WhatsApp and Facebook Messenger. These tools were used in the course of the Research Seminar in the School of Social Work, identifying its usefulness during the research process, demonstrating that they were useful in the collection, capture and analysis of the information.

Keywords: Data Collection; Technologies; Social Work.

\footnotetext{
${ }^{1}$ Trabajadora Social, Universidad de Colima. Doctora en Estudios Latinoamericanos, Universidad de Tulane, Estados Unidos. Profesora de tiempo completo de la Facultad de Trabajo Social, Colima, México. Correspondencia dirigirla a: preciado@ucol.mx
} 
Retos para el trabajo de campo, instrumentos tradicionales en modalidades actuales para el trabajo social por Susana Aurelia Preciado Jiménez. http://doi.org/10.29035/pai.4.41

\section{Introducción}

La formación profesional en Trabajo Social, como muchas otras, se va transformando día a día, considerando que además de actualizar los contenidos de las asignaturas, también se incorporan nuevas bibliografías, así como los que surgen a partir de un proceso de reestructuración, resultado de una revisión, a partir del Modelo Educativo que impera actualmente en la Universidad de Colima, para la formación de trabajadores sociales a nivel licenciatura, así como cambios que se suscitan por recomendaciones de organismos evaluadores externos. De igual manera, se consideran los resultados que ofrecen los estudios de pertinencia y factibilidad, lo que conlleva a convertir nuevas necesidades en asignaturas distintas, o bien mismas asignaturas con modalidades de aprendizaje diferentes.

Por tanto, existen transformaciones que deben hacerse por la esencia misma de un proceso de formación, así como también las que son inherentes a la especificidad de la profesión; en este caso, es incluir los requerimientos para la formación de trabajadores sociales, en donde la innovación debe estar implícita, es decir, preparar a los profesionistas a afrontar e intervenir ante necesidades sociales que pueden ser manifestaciones individuales, de grupos o comunidades, o bien, la intervención o investigación de fenómenos socio familiares, que son el resultado de procesos socioculturales que aun cuando no existían en los contenidos en el momento en que se organizó el currículo, por su importancia quienes participan en el proceso de enseñanza-aprendizaje deben incluirlos.

Precisamente, partiendo de este último aspecto, en este artículo se presenta la manera cómo se ha resuelto el tener que recolectar información de fuentes primarias en un contexto sociocultural que no facilita el intercambio de información cara a cara; por lo tanto, se tuvo que implementar estrategias diferentes incorporando las Tecnologías de Información y Comunicación (TIC) y las redes sociales, que aun cuando no fueron revisadas en clase, por no corresponder a la materia, sí se utilizan como parte de esas herramientas de uso común entre los estudiantes para otros escenarios de su vida.

En este sentido, los estudiantes de Trabajo Social y muy probablemente de otras disciplinas, han visto como en los últimos años los procesos de enseñanzaaprendizaje han incluido el uso de las TIC y de las redes sociales; inclusive se han diseñado cursos de capacitación ex profeso. De igual manera, se han hecho estudios del uso de las TIC y las Redes Sociales en los diferentes ámbitos de la vida, a partir de diferentes grupos poblacionales, encontrándose ventajas y desventajas. Sin embargo, no se ha reflexionado sobre que el uso de esas tecnologías o plataformas también pueden ser útiles como nuevos instrumentos para la recolección de información de primera mano, centrándose más en la aplicación de estas tecnologías en el salón de clases, considerando que al adquirir una competencia tecnológica, el estudiante puede adquirir conocimientos, desarrollar habilidades y actitudes e, incluso, adoptar un rol dentro de su proceso 
de formación en la universidad, independientemente de su formación disciplinar, sin considerar que también esas plataformas y/o aplicaciones se pueden convertir en aliados para la recolección de datos de fuentes primarias.

Sin duda, el uso de las TIC, así como las tecnologías del aprendizaje y el conocimiento (TAC), son un reto tanto para docentes como para los propios estudiantes. Para el docente, su utilización en clase requiere de un proceso de aprendizaje para su implementación; para el estudiante, debe comprender que el uso de estos dispositivos en clase, se convierten en herramientas que le van a permitir, compartir, descubrir, generar, aprender, establecer canales de comunicación con su entorno educativo y, por tanto, deberá desarrollar habilidades para el uso de estos e incorporar como propósito para su formación profesional.

Lo indicado anteriormente ha sido analizado por Nakano Osores et al. (2013) cuando señalan que al inicio del siglo XXI "han emergido nuevos espacios de socialización mediados por las TIC" (p. 137), modificando con ello la manera en que se interactúa en el salón de clase, al tener que desarrollar habilidades digitales, tanto por parte del docente como por el estudiante, para utilizarse como instrumentos o medios de interacción en el proceso de enseñanza-aprendizaje.

Por ello, bajo este escenario, y ante la incorporación en las universidades, en los cursos de formación docente sobre el uso de las TIC, del uso de entornos virtuales y, por otro lado, observando que cada día los estudiantes utilizan como medio de interacción los dispositivos móviles, se hizo una propuesta en la materia de Seminario de Investigación en la Facultad de Trabajo Social, para que algunas TIC e incluso Redes Sociales mediadas por el internet, pudieran considerarse como instrumentos para la recolección de datos, considerando sus implicaciones éticas, en el grupo de sujetos sociales interesados en participar en los proyectos de investigación, así como también que, al contar con esa información, puede ser analizada y ofrecer respuestas a sus objetivos y preguntas de investigación.

En un estudio, realizado por Tuñez-López y Sixto-García (2012), se encontró que las redes sociales pueden convertirse en un complemento de la docencia; incluso lo mencionan como un espacio suplementario de apoyo entre las diversas interacciones entre docente y estudiante, pero que no sustituye al proceso de enseñanza-aprendizaje. En este mismo sentido, Brito y Toloza (2012) mencionan que las redes sociales pueden ser un complemento para el desarrollo del ámbito y de los entornos actuales de aprendizaje; además de que su uso no es solamente una moda, sino que estas se han convertido en patrones de comunicación, por lo que debe realizarse una análisis crítico y reflexivo sobre trascendencia y efectividad dentro del salón de clases.

Aunado a ello, es menester citar a Duderstand (1997) quien muestra cuatro escenarios, donde visibiliza el uso de las TIC en este tiempo: a) La importancia 
del conocimiento como un factor clave para determinar seguridad, prosperidad y calidad de vida; b) La naturaleza global de nuestra sociedad; c) La facilidad con la que la tecnología -ordenadores, telecomunicaciones y multimedia- posibilita el rápido intercambio de información; y d) El grado con el que la colaboración informal (sobre todo a través de redes) entre individuos e instituciones está reemplazando a estructuras sociales más formales, como corporaciones, universidades, gobiernos.

Es así que, bajo esas consideraciones y el propio contexto sociocultural que impera en México, se observó que la manera en que los estudiantes en la materia de Seminario de Investigación comenzaron a acercarse a la información de fuentes primarias cada día se tornaba complicada y, en principio, se pensó que podría ser por el tipo de proyectos que se presentaron tales como bullying, trastornos alimenticios, discriminación, violencia, familias disfuncionales; empero esto también se encontró en temas que hacen referencia a familias migrantes, estudios sobre las preferencias de estudios, o la trayectoria profesional, el síndrome del burnout entre docentes, inclusión de temas emergentes en el trabajo social, porque los principales obstáculos que se documentaron al momento de la recogida de la información fueron: a) la disponibilidad del tiempo, tanto para los entrevistados como por los entrevistadores; b) la exposición de ciertos temas incluidos en la entrevista, que hicieron sentir a los entrevistados observados y se mostraban cohibidos, con respuestas cortas, e incluso omitían las respuestas; c) interés por los entrevistados de contar con anticipación del instrumento (señalando que requerían saber de lo que se trataría la entrevista) y, al llegar al día de la entrevista, se encontraron con el documento contestado y con ello se daba por concluida la visita; d) la incertidumbre de la población ante el uso de su información, generando problemas de seguridad y confianza por parte de la ciudadanía, así como de las propias instituciones con el manejo de los datos, entre otras situaciones.

Ante esto, por supuesto que se cuestionó ¿qué estaba sucediendo en nuestro contexto? y ¿cómo podríamos resolverlo para poder realizar las encuestas, las entrevistas en profundidad e incluso los grupos de discusión?, ¿Qué se tenía que hacer para ofrecerles confianza a los entrevistados con referencia al resguardo de la información, o bien de la identidad de los participantes?

Es así que este artículo reconoce la necesidad de incorporar en la materia de investigación el uso de las TIC, particularmente durante la recopilación de la información de campo, para lo cual se tuvo que proponer estrategias de acercamiento a la población, generar el espacio del rapport y ofrecerles seguridad; por otro lado, se encontró que los docentes requieren capacitación para identificar que las TIC pueden ser utilizadas como instrumentos de comunicación para la recopilación de información, el trabajo colaborativo mediante el uso de aplicaciones en red (drive, nube, documentos en red, formularios, etc.), sin descuidar aspectos relacionados con las destrezas emocionales e intelectuales de los estudiantes, y 
motivarlos a incorporarse a un escenario de flexibilidad, en donde se seguirían utilizando las técnicas e instrumentos de recolección ya existentes; sin embargo, la modalidad de acercamiento a la población sería mediada por las TIC.

\section{Misma herramienta, diferente forma de interacción}

Hoy día, la forma de interactuar con las personas parece estar modificándose, y las redes sociales comienzan a tener mayor peso en la manera de socializar entre las personas. En la última década, se ha escrito sobre las redes sociales como una herramienta poderosa que puede influir positivamente en la sociedad (Castillo-Ríos, 2013), incluso se les ha considerado como un medio de organización rápido, eficaz y barato; sin embargo, también se reconoce que pueden afectar negativamente el comportamiento e inclusive el rendimiento de las personas cuando estas no son utilizadas de manera adecuada.

En tales circunstancias, es válido revisar el potencial que tiene el uso de las TIC, particularmente cuando se pretende generar nuevos espacios para la recolección de datos de primera mano, situación que puede capitalizarse al utilizarlas como un medio para la aplicación de técnicas de recolección utilizando los instrumentos de encuestas, grupos de discusión y entrevistas en profundidad. Así mismo, al reconocer que existen espacios de interacción a partir del uso de las TIC, y que se pueden generar espacios virtuales para el desarrollo de las técnicas antes señaladas.

Como se comentó anteriormente, los profesores que imparten la materia de Seminario de Investigación detectaron que los estudiantes manifestaban cada día tener menos acceso a información, por lo que se expuso en clase la situación y los comentarios de los estudiantes se centraron en buscar propuestas que permitieran recolectar información de fuentes primarias, pero haciéndolo bajo otros esquemas; es decir, hacerse igual pero diferente, dado que parecía que, en algunas investigaciones, la aplicación de la entrevista cara a cara se estaba convirtiendo en una barrera. Fue así que se inició una búsqueda y utilización de diversas técnicas de recolección de información, a partir de la incorporación en los talleres de discusión, de reflexión o de capacitación, preguntas que recogieran datos útiles para el área de las ciencias sociales y humanidades, en particular para Trabajo Social. También se incursionó en el uso de la técnica narrativa, utilizándose a partir de la generación de cuentos, cartas o, incluso, de dibujos, los cuales se saben generan una gran cantidad de información cualitativa.

En esa etapa de búsqueda y aplicación de otras formas de recopilar información, se encontró un estudio realizado en la Universidad de Málaga, en donde al alumnado se le aplicó un cuestionario online para realizar un estudio de estilos de vida (Sánchez-Vega, 2012), tomándose como punto de partida que 
la mayoría de los jóvenes inscritos en la institución escolar contaban con un teléfono móvil, o bien que estaban expuestos a una forma de tecnología como la televisión, computadoras e internet, partiendo de la premisa de que las TIC son un componente importante en la conexión con el mundo así como con las personas que son parte del círculo social y con quienes se pretendió comunicarse. Por tanto, considerando las ventajas y desventajas de las TIC y bajo esas premisas, se hizo un estudio, usando el cuestionario tradicional, utilizando el formulario de Google y enviándolo por correo electrónico. En los comentarios de los investigadores, se señala que el uso de esta herramienta les generó un decremento en los costos al aplicar el instrumento, una mayor cantidad de sujetos en la muestra, puesto que no se requiere de conocimientos especiales para responder el instrumento, tan solo contar con una cuenta de internet; por otro lado, los datos obtenidos pudieron analizarse en el paquete estadístico SPSS así como un análisis factorial para comprobar el rigor científico del cuestionario, además de conocer los estilos de vida de los jóvenes universitarios.

Con base en esos resultados, se consideró pertinente hacer una propuesta a los estudiantes de la materia de Seminario de Investigación II para utilizar las TIC en la recolección de datos de sus proyectos de investigación, y las actividades desarrolladas se están sistematizando para conocer los resultados, la veracidad de los mismos, y hacer los análisis requeridos para identificar las áreas de oportunidad que se encontraron a partir de utilizar esta propuesta.

\section{Método}

En el desarrollo de este trabajo se empleó una metodología cualitativa, a partir de la revisión de documentos de investigación elaborados en la materia de Seminario de Investigación de los años 2016-2017, en donde se incluyó el uso de las TIC como una modalidad para la recopilación de datos. Para el análisis e interpretación de los datos se utilizó la Teoría Fundamentada, a partir de la generación de tres categorías: "medio de obtención de la información", "tipo de instrumentos", y "Resultados", que surgieron a partir de la implementación de cinco proyectos de investigación, en donde los medios de obtención de la información fueron a través del "formulario de Google", "Grupo de Facebook" y "Grupo de WhatsApp".

El muestreo consitió, entonces, en cinco trabajos de investigación realizados por estudiantes, en donde los sujetos de investigación accedieron a utilizar las TIC; los sujetos de investigación en esos proyectos fueron estudiantes de nivel medio superior-preparatoria (87) y superior-licenciatura (29), egresados de la licenciatura en Trabajo Social (18), docentes de educación superior (225) y personas de la comunidad (6). Las modalidades para la aplicación de los instrumentos fueron: 
Formulario de Google (cuestionario y entrevista estructurada), grupo de Facebook (entrevista descriptiva), grupo de WhatsApp (entrevista inicial).

El objetivo en este proyecto fue generar una propuesta para la recolección de datos de fuentes primarias utilizando las herramientas que se encuentran en la red, tanto en Google como en las redes sociales. Y como objetivos específicos, a) identificar las ventajas y desventajas de las TIC para obtener información de fuentes primarias; b) identificar el uso de las TIC en la investigación, particularmente, en la recolección de información de fuentes primarias, así como en el análisis de los resultados; y, c) presentar una propuesta sobre el uso de las TIC considerando los aspectos éticos que implica realizar una investigación social.

\section{Resultados}

El Trabajo Social contemporáneo se enfrenta a retos en el proceso del trabajo de campo, particularmente en la recolección de los datos; por ello, a partir de que se observó la existencia de contratiempos en la recolección de información cara a cara de algunos proyectos en la materia de Seminario de Investigación, se propuso unas estrategias para utilizar los mismos instrumentos de recolección de datos (cuestionario y entrevista) bajo nuevas modalidades: Formulario de Google y los grupos de Facebook y WhatsApp, permitiendo una aplicación única y rápida (en el caso de los cuestionarios), disminuyendo costos, logrando capturar la información tal y como fue expresada por el entrevistado (entrevistas) y contando con la información transcrita para el análisis de datos y que esta información pueda ser portable a los programas identificados para ello.

El proceso de la aplicación de los instrumentos descritos en los trabajos favoreció identificar tres aspectos importantes en el proceso metodológico.

\section{Categoría "medio de obtención de la información"}

El formulario de Google (cuestionario y entrevista estructurada) fue utilizado por cuatro proyectos de investigación, tres donde la investigación fue cuantitativa y los sujetos de investigación fueron estudiantes de preparatoria y licenciatura, así como un grupo de docentes de educación superior; y uno cualitativo donde los sujetos fueron egresados de la licenciatura en Trabajo Social. A los grupos de personas de los estudios que utilizaron el cuestionario, se solicitó autorización en la institución educativa, y se permitió ingresar a los grupos de estudiantes para hacerles una invitación in situ, para el caso de los docentes, en la oficina de formación docente, enviaron un correo electrónico a los profesores invitándoles a participar, y señalando que era una investigación por estudiantes de la Facultad de 
Trabajo Social. En los tres casos, se les comunicó el objetivo de las investigaciones, y se les dio a conocer el enlace al formulario de Google, para que se pudieran dar de alta y responder el mismo. Los participantes fueron respondiendo las preguntas que se les hicieron. Al término del cuestionario, se les envió un correo de agradecimiento por su participación. El tiempo de respuesta que se obtuvo en los tres casos fue muy rápido (dos semanas), además, las respuestas fueron entregadas por la misma aplicación en una base de datos de Excel, en donde también se lograron obtener resultados preliminares. Esta base de datos pudo ser exportada al SPSS y se observó que no había errores de captura, y pudieron realizarse los análisis pertinentes de acuerdo al tipo de investigación sin contratiempos.

En lo que respecta a la entrevista estructurada para el grupo de egresados de Trabajo Social, se realizó primero un grupo de WhatsApp, y de ahí se les invitó a responder la entrevista estructurada, utilizando el enlace generado en el formulario de Google. Por tanto, también se contó con la información transcrita en tiempo y forma, respetando la manera en que cada uno de ellos se expresó.

La otra modalidad fue el grupo de Facebook, utilizada por un equipo de investigación con personas de la comunidad; aquí la muestra fue voluntaria, el trabajo fue cualitativo. En principio, las estudiantes realizaron un cartel donde se describió la investigación y, al colocarlo en su muro de Facebook, pudieron verlo sus "amigos" y los "amigos de amigos". Se les pidió que quienes mostraran interés mandaran un mensaje privado, para poder conformar un grupo. En el grupo inicial, se postearon algunas preguntas detonadoras, que fueron respondidas por los participantes, en los tiempos en que cada uno accedían a la red (asincrónica), encontrándose que el ambiente en el grupo fue horizontal, se evitaron las censuras y hubo una comunicación fluida. Cabe señalar que las impresiones de los integrantes del grupo señalaron que fue cómodo y se tuvo una alta participación. En el grupo se estableció el rapport a través de un diálogo abierto, y con algunas preguntas que les fueron llevando al tema. Después se les invitó a participar en una entrevista individual por ese mismo medio, utilizando el chat de la red, en donde cada uno de ellos tuvo la oportunidad de expresar sus opiniones con respecto al tema de investigación. Una de las ventajas que comentaron las estudiantes fue que lograron recolectar información valiosa y que, además, fue transcrita tal cual fue expresada por los participantes, lo que les permitió realizar su análisis cualitativo de manera adecuada y en un tiempo "sorprendentemente rápido" (entrevistadora)

En el grupo que se utilizó el WhatsApp fue con egresadas de la licenciatura. Para ello se obtuvo acceso a un grupo de egresadas, se les invitó a participar en la investigación y, con aquellas personas que accedieron, se creó un grupo para la investigación. En el grupo primero se hicieron tres preguntas detonadoras, las cuales les sirvió para reestructurar su entrevista, y después se les pidió que accedieran al formulario de Google para que la respondieran. La estudiante 
responsable de este proyecto manifestó que contó con la información total, tanto de la entrevista inicial como de la estructurada, estuvo transcrita y respetaron la forma en que cada persona se expresó, lo que le ayudó para realizar el análisis cualitativo propuesto.

En las tres modalidades expresaron los responsables de los proyectos que algo que les pareció muy interesante fue que todo el tiempo pudieron hacer un seguimiento de la aplicación de sus instrumentos; los tiempos de los participantes se respetó, además tanto en el Facebook como en el WhatsApp lograron observar sus emociones, dado que los participantes hicieron uso de los emoticons.

\section{Categoría "tipo de instrumentos"}

Los instrumentos utilizados fueron los que se conocen tanto en las investigaciones cuantitativas o cualitativas, y se utilizó un proceso en su diseño, validación y aplicación, utilizando de igual forma las TIC, además de que se capacitaron para poder convertirse en los administradores de sus grupos.

El diseño de los instrumentos de recolección de datos se realizó a partir de contar con una propuesta del cuestionario o entrevista, se llevó a cabo un pilotaje con una muestra de personas con características similares a la población tipo de su investigación, y la validación por un grupo de profesores universitarios expertos en ese tema; la diferencia en estos proyectos es que los responsables de los proyectos utilizaron las TIC para su implementación también, con lo cual se midió también la efectividad de la modalidad. Por supuesto que en los instrumentos se verificó su pertinencia conforme a los objetivos de investigación y a su pregunta de investigación (los trabajos que formaron parte de la muestra fueron de tipo exploratorio), a continuación se describe la manera en que se realizaron cada uno de ellos.

El cuestionario se diseñó utilizando los objetivos de la investigación, generando las variables y categorías. El instrumento se subdividió, de acuerdo al estudio, en diversos bloques o secciones, a través de los cuales se engloba lo señalado en sus objetivos de investigación. El cuestionario fue piloteado con una muestra similar a la que se aplicaría el estudio, haciéndoles la invitación a participar, se les proporcionó el enlace del formulario y ahí lo respondieron, dejándoles además un espacio para comentarios de mejora para cada uno de los ítems. De igual manera se validó el instrumento con expertos en el tema, a quienes se les pidió revisar el documento, e hicieron sus observaciones por ítem. Se rehicieron los instrumentos y se asistió a espacios destinados para la investigación para poder contar con los permisos para ello. 
En el caso de las entrevistas también se hizo el proceso de pilotaje/validación de los instrumentos con casos similares a la muestra y con expertos. La relación empática y el rapport necesarios en una entrevista se estableció a partir de establecer esa conexión entre las partes, y compartir información que tal vez parece irrelevante a la investigación al principio; sin embargo, es crucial para la transmisión de datos importantes posteriormente.

Por tanto, cuando se utilizan las opciones que se ofrecen por las redes sociales y las aplicaciones de Google para recolectar información de campo, es crucial que se establezca la relación empática o el rapport con los participantes, y tanto en el Facebook como en el WhatsApp se tuvieron opciones para establecer lazos afectivos y vínculos que fortalecieron una mejor comunicación. Sin embargo, es importante reconocer que, en ocasiones, el uso de las redes sociales puede ser un tanto desinhibidor para el entrevistado, al no verse con su entrevistador, y el entrevistador puede sentirse rebasado por esa misma situación por lo que debe estar habilitado para ello. Manifestaron las responsables de los proyectos que cuando estuvieron en línea con sus entrevistados se logró una comunicación efectiva, y hubo quienes incorporaron imágenes como los Emoticonos, con los cuales se podían expresar emociones y otorgarle emotividad al ejercicio. Sin embargo, también pueden existir desventajas, como que el entrevistador utilice ese espacio para hacer un ejercicio de catarsis, o que algunas preguntas las responden de manera muy breve, y podría generar angustia en las entrevistadas al sentir que no se lograba profundizar en el tema. Lo que sí fue manifestado por las estudiantes que utilizaron la entrevista fue que se requiere tener más formación en el manejo de la técnica.

\section{Categoría "Resultados"}

Los resultados se dividieron en ventajas y desventajas con el uso de las TIC para la recolección de datos, en los momentos del proceso aprendizaje, para los entrevistados y para los entrevistadores. A continuación, se expone un resumen de ventajas y desventajas:

Ventajas en el proceso de enseñanza aprendizaje:

- Motivación en el tema de investigación

- Desarrollo de la iniciativa

- Aprendizaje cooperativo

- Alfabetización digital y audiovisual

- Mejora de las competencias de expresión y creatividad

- Búsqueda de información relacionada con su tema de investigación 
Para los entrevistados

- Atractivo

- Acceso a la información en sus propios tiempos y ritmos

- Flexibilidad

- Colaborativo

- Respeto a su tiempo

Para los entrevistadores

- Fuente de recursos para la recolección de datos

- Tratamiento de datos de manera individual de acuerdo a la técnica y el programa para su análisis

- Mayor contacto con los entrevistados sin interrumpir en sus tiempos

- Mayor control de la información

Desventajas en el proceso de enseñanza-aprendizaje

- Dispersión (querer abarcar mucha información)

- Diálogos rígidos o sin contenido al inicio

- Visión parcial de la realidad

- Ansiedad

Para los entrevistados

- Inversión de tiempo

- Sensación de desbordamiento

- Falta de conocimiento de algunos temas y no saber cómo responder

Para los entrevistadores

- Estrés

- Desfases respecto a otras actividades

- Sentir que no profundizaban en el tema (entrevistas).

- Requieren tener más formación en el manejo de la técnica

- Actualización en el uso de las aplicaciones como un recurso educativo 
Por tanto, entre las ventajas se logró observar que el realizar su trabajo de esta manera fue una experiencia satisfactoria, aun cuando también los responsables de los proyectos expresaron sentimientos encontrados. Por ejemplo, cuando tenían la oportunidad de observar en los mensajes del chat o del grupo que crearon para la entrevista inicial, se presentó igual que una conversación con una amigo en el chat, al encontrar que los mensajes se iban entremezclando y, por momentos, el entrevistador se perdía en toda esa inmensidad de información; o bien cuando tuvieron varias intervenciones, y al principio no lograban comprender lo que ahí se dijo, pero que al final, cuando se da lectura, se puede observar el hilo conductor; también señalaron que en esas conversaciones se lograba sentir la emoción, e incluso se pueden imaginar las reacciones ante ciertos comentarios.

Otra ventaja fue que, durante el proceso de investigación, con el uso de las TIC se puede evitar el sesgo al momento de la captura, dado que, tanto en las preguntas abiertas como en las entrevistas, el modo de comunicación fue la escritura, es decir, se logró contar con la transcripción fiel de lo que expresó el entrevistado. Por otro lado, el entrevistado tuvo la opción de borrar, o corregir lo que ha visto escrito. Por tanto, errores de transcripción o de interpretación fueron evitados con este medio y, por supuesto, también existe un ahorro de tiempo que permite agendar nuevos encuentros para profundizar en algunos aspectos relevantes.

También se encontraron algunos obstáculos, al utilizar las TIC, y fue en algunas entrevistas que, precisamente por tener que ser escrita por el entrevistado, al inicio las respuestas fueron cortas; sin embargo, conforme el entrevistado se fue sintiendo cómodo con las preguntas, o con el tema, se logró observar mayor fluidez en sus transcripciones. El rol del entrevistador consiste en hacerle sentir cómodo, que no tenga preocupaciones por la ortografía o la forma en que escribe, sino que lo importante es el mensaje que comparte.

Otra desventaja que se observó fue cuando uno de los participantes se sintió incómodo y abandonó el canal de la conversación y, por tanto, su participación quedó interrumpida hasta no saber las razones por las cuales abandonó el grupo. Sin embargo, esto no solamente es una situación que sucede en este tipo de entrevista, pues igual en la que se realiza cara a cara la persona puede desistir de continuar participando.

En los responsables de los proyectos de investigación se observó que:

- Al estar familiarizados con el uso de la computadora y de las redes sociales, expresaron un cierto grado de frustración cuando identificaron que requerían mayores herramientas para utilizarlas, empero una vez rebasado ese periodo, la emoción que mostraron al comenzar a obtener información y que fue respondiendo sus objetivos, se observaron satisfechos. 
- La presentación y la elaboración de su trabajo se hizo a partir de su equipo de cómputo o del que les fue asignado en la facultad, y siempre estaban conectados al correo electrónico, pues utilizaron la herramienta de Documentos en Google, así que al momento de tener dudas se conectaban con el docente para buscar responderlas y continuar con sus tareas en el mismo espacio, sin tener que dejar de hacer lo que estaban haciendo para movilizarse. Esto generó al principio frustración tanto en los estudiantes como docentes, pues no tenía'n la respuesta de manera inmediata unos, y los otros no podían siempre responder al momento. Por lo que se tuvo que establecer tiempos y saber utilizar la ventana de "añadir comentario" y esperar a que se tuviera la respuesta. Por otro lado, también generó temor en los asesores, quienes tuvieron que aprender esa forma de trabajo colaborativo en red y todas las ayudas que se ofrecen.

- Los estudiantes reconocieron que, al utilizar el formulario de Google, al mismo tiempo obtuvieron la base de datos en Excel, incluso tuvieron algunos resultados preliminares. Eso les facilitó la captura de la información (que no la hicieron) y poder explorar formas de analizar en la misma aplicación de Formularios de Google, y presentar su información les resultó interesante, y al mismo tiempo sintieron que el tiempo de captura y de presentación de los resultados se redujo en comparación de sus compañeros que hicieron el trabajo en la forma tradicional. En los asesores también se observó satisfacción al poder visualizar el avance tanto de la información como de la manera en que los estudiantes interactuaron con los datos; también lograban visualizar que los cambios propuestos se realizaban, y no se perdían entre lo que les había sugerido y si lo habían realizado o no.

- Otro elemento que causó mucha alegría en los estudiantes fue que podían cerciorarse de que su trabajo siempre estaba disponible, pues lo había guardado en la nube, así que podían visualizarlo en cualquier dispositivo siempre y cuando tuvieran conexión de red, y eso les aseguró no tener pérdidas de sus documentos, llevar un seguimiento de sus observaciones al compartir su trabajo con su asesor o profesora de la materia, lo que les generó mayor seguridad y podía ir haciendo su trabajo de manera más profesional.

\section{Discusión y conclusiones}

La propuesta de mostrar algunos de los retos que enfrentan los estudiantes en la recolección de datos de investigación no es ajena a los investigadores sociales; por ello, el reconocer que se pueden utilizar los instrumentos tradicionales como el cuestionario, la entrevista, los grupos de discusión con modalidades que ofrece las redes sociales como el Facebook, el WhatsApp, o los formularios de 
Google e identificar sus aciertos y obstáculos, permite reconocer que las redes sociales puede ser útiles para el acercamiento con grupos poblaciones y obtener información de fuentes primarias, es decir, lograr un contacto cara a cara a través de las TIC.

De igual manera, reconocer que la información obtenida por este medio nos obliga a cumplir con los aspectos éticos que implica realizar una investigación social, logrando que el utilizar las herramientas que ofrece el Google permite a los participantes mantener su anonimato, proteger su identidad; pero de igual manera, a quienes realizan la tarea de recopilación de información, reconocer que se debe tener un uso ético de los datos obtenidos, y poder dar a conocer los resultados en los espacios para los cuales fueron pensados e informados a los participantes.

Por ello, se advierte que el uso de las TIC y las redes sociales en Trabajo Social, nos permite tener una gama amplia de posibilidades en la investigación social, y de igual manera nos exige adquirir las habilidades y competencias para hacer un uso eficiente y efectivo de las mismas.

Algunos retos o temas que se puede incursionar o profundizar a partir de este trabajo son:

- Hacer una comparación entre investigaciones que utilizan la modalidad cara a cara y la que utiliza la comunicación mediada por las TIC para identificar las similitudes y diferencias entre los datos obtenidos.

- Sistematizar la habilidad del investigador en estos espacios que proporcionan las redes sociales para generar la "confianza" y cómo podría verse afectada por el uso de estos canales de comunicación.

- Realizar un estudio comparativo entre procesos de investigación cualitativa y cuantitativa en cuanto a sus retos, manejo de la información y sobre la comunicación no verbal. 


\section{Referencias}

Brito, J. G., y Toloza, E. A. (2012). El uso de redes sociales por parte de las universidades a nivel institucional. Un estudio comparativo. Revista de Educación a Distancia, 1-38.

Castillo-Ríos, R. A. (2013). Ventajas y desventajas del uso de las redes sociales en el estudio universitario de alumnos de antiguo y nuevo ingreso de la Universidad Francisco Gavidia (UFG) Sede San Salvador. San Salvador: Universidad Francisco Gavidia.

Duderstand, J. (1997). The future of the university in an age of knowledge. Journal of Asynchronous Learning Networks, 78-88.

Nakano Osores, T., Águeda Chávez, M., Begazo Ruíz, J., Garret Vargas, P., Velasco Tapia, A., y Rosales Lam, A. M. (2013). Uso de tablets en la educación superior: una experiencia con iPads. Digital Education Review, 24, 135-161.

Sánchez-Vega, E. (2012). Las TIC como medio de investigación y evaluación en un estudio sobre estilos de vida. En Evaluación por competencias mediante eRúbricas. Málaga: Universidad de Málaga. Obtenido de http://gtea.uma.es/ congresos/CDROM/

Tuñez-López, M., y Sixto-García, J. (2012). Las redes sociales como entorno docente: análisis del uso de Facebook en la docencia universitaria. Píxel-Bit: Revista de Medios y Educación, 77-92. 


\section{Como citar este artículo}

Preciado, S. (2018). Retos para el trabajo de campo, instrumentos tradicionales en modalidades actuales para el trabajo social. Revista Pensamiento y Acción Interdisciplinaria, 4(1), 41-55. DOI: http://doi.org/10.29035/pai.4.1.41

\section{Dirección de correspondencia}

Susana Aurelia Preciado Jiménez

Doctora en Estudios Latinoamericanos.

Profesora de tiempo completo de la Facultad de Trabajo Social, Universidad de Colima, México.

Contacto:

preciado@ucol.mx

Recibido: $24 / 07 / 2018$

Aceptado: 25/09/2018 